\title{
Measuring the stop point of rapid smoking
}

\author{
MURRAY E. JARVIK, MARTIN R. ROSENBLATT, CATHERINE L. CARPENTER, \\ NICHOLAS H. CASKEY, TODD M. GROSS, and WILLIAM J. MCCARTHY \\ West Los Angeles V.A. Medical Center, Los Angeles, California \\ and University of California, Los Angeles, Los Angeles, California
}

\begin{abstract}
A method and an apparatus are described for measuring the point at which subjects elect to stop smoking when cigarette smoke is delivered more rapidly than in typical smoking. The device allows either the subject or the experimenter to control the volume of smoke and the interval between puffs. The device delivers reliable quantities of particulate matter from cigarette smoke. Subjects retested a week apart exhibited consistent stopping points.
\end{abstract}

Cigarette-smoking behavior, like other consummatory processes, has homeostatically controlled upper and lower boundaries (Kozlowski \& Herman, 1984). Many studies have shown that smokers will titrate their smoke intake to keep blood nicotine levels and, presumably, brain levels fairly constant (Benowitz, Jacob, Denaro, \& Jenkins, 1991; Kozlowski \& Herman, 1984; Russell, 1989). The present paper illustrates a method for measuring the highest volume of smoke that subjects are willing to inhale with controlled smoking. Smokers seek a minimum level of nicotine and other smoke constituents for satisfaction, and they avoid a maximum level that produces disagreeable sensations. As smoking novices usually discover quickly, smoking irritates the throat and can produce unpleasant dizziness and nausea, which causes novices to stop. More experienced smokers pace their smoking to avoid these symptoms, but rapid smoking can reinstate this aversion. Rapid smoking has been used as an aversive conditioning technique to aid in smoking cessation (Glasgow, Lichtenstein, Beaver, \& O'Neill, 1981; Sachs, Hall, Pechacek, \& Fitzgerald, 1979).

The negative feedback mechanisms that control tobacco smoking prevent nicotine poisoning and potentially lethal nicotine overdose. The protective cues involved are both peripheral and central, since nicotine activates sensory receptors in the oropharynx and upper airway, the carotid artery, and the brain. By contrast, substances like inhaled cocaine ("crack") have such high ceilings of aversive feedback that many individuals cannot stop self-administration and may even kill themselves with an overdose (Johanson and Fischman, 1989).

To measure the maximally tolerated dose of smoke, we constructed a relatively simple cigarette-smoking mechanism that offers precise control of the volume and timing of smoke inhaled. This is a simplified modification

Preparation of this article was supported in part by the California Tobacco-Related Disease Program (Grant IRT 87) and the NIDA (Grant 5 R01 DAO 5111). The assistance of George Mazzei is gratefully acknowledged. Correspondence should be addressed to M. E. Jarvik, Psychopharmacology Unit, Brentwood, V.A. Hospital, Los Angeles, CA 90073. of devices described by Gilbert, Jensen, and Meliska (1988); Hatsukami et al. (1990); C. S. Pomerleau, Majchrzak, and O. F. Pomerleau (1989); O. F. Pomerleau, C. S. Pomerleau, and Rose (1989); O. F. Pomerleau, Rose, C. S. Pomerleau, and Majchrzak (1989); and Rose, Tashkin, Ertle, Zinzer, and Lafer (1985). These systems will deliver known quantities of cigarette smoke to subjects (C. S. Pomerleau et al., 1989; O. F. Pomerleau, C. S. Pomerleau, \& Rose, 1989; O. F. Pomerleau, Rose, et al., 1989). In one ingeniously contrived system (Levin, Rose, \& Behm, 1989), the subject actually draws the smoke directly from a cigarette and displaces air in a syringe distal to the cigarette. However, because the cigarette is totally enclosed, replacing it is rather demanding. We designed an apparatus to allow either the experimenter or the subject to control the volume or timing of cigarette-smoke puffs. By extrapolating from these values, we may estimate the total daily intake of toxic substances that may result in tobacco-related diseases.

To test the reliability of the apparatus, we measured the maximal smoke tolerance by a modified rapid-smoking procedure. We assumed that smokers forced to puff more rapidly than usual would accumulate nicotine at a greater rate than is customary. Thus, they would find the smoke sensations, both peripheral and central, changing from pleasant to unpleasant. Finally, these sensations would become so unpleasant that further smoking would be unacceptable. We called the point at which they refused to continue smoking the "stop point."

\section{METHOD}

\section{Apparatus}

As can be seen in Figure 1, the centerpiece of the apparatus is a hand-ground $100-\mathrm{cc}$ syringe that functions as a smoke piston pump and reservoir. It is attached to a 9-mm-diameter mouthpiece, approximately the same diameter as a commercially available cigarette $(8 \mathrm{~mm})$, connected to a 2-cm-diameter one-way flap valve that allows passage of air or smoke in only one direction, towards the mouth. An electric pneumatic valve (Skinner Electric) is situated between the syringe and the cigarette to 


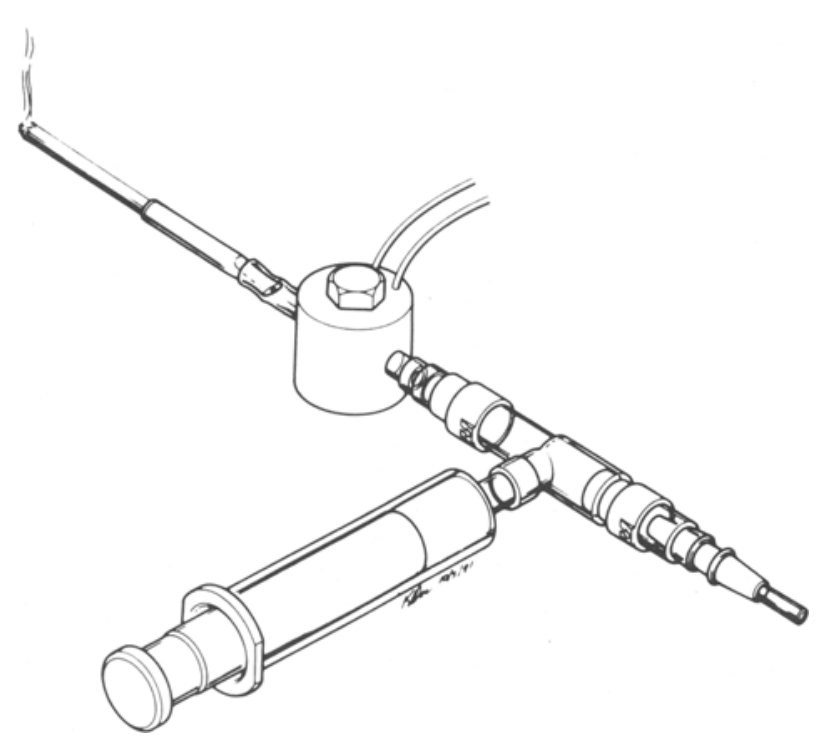

Figure 1. The syringe piston pump used in this study. A fixed volume of smoke is drawn into the syringe by the experimenter and then inhaled by the subject.

prevent any movement of smoke until the experimenter or timer opens the valve.

\section{Subjects}

Eight male subjects, all heavy smokers, were recruited from inpatient programs at the West Los Angeles V.A. Medical Center. All were veterans and of lower socioeconomic background. For this sample $(N=8)$, the mean number of reported cigarettes smoked per day was 20.8 $(S D=3.4)$; the mean self-reported depth of inhalation was $2.6(S D=0.5)$ on a 4-point scale, with $0=$ no inhalation and $4=$ very deep inhalation; and the mean Fagerstrom dependence score (Fagerstrom \& Schneider, 1989) was $7.6(S D=1.2)$.

\section{Procedure}

To calibrate smoke delivery with this apparatus, Cambridge filters were placed in the mouthpiece. The experimenter injected five 40-cc puffs of smoke through previously weighed filters, which were then reweighed to measure the total particulate matter (TPM) delivery. This procedure was repeated twice for each of five cigarettes. Two different brands of commercially available cigarettes, Marlboro king-size soft pack and Carlton ultralow tar/nicotine, were compared using the same procedure.

During experimental sessions, the subject sat in front of the mouthpiece and waited while the experimenter placed a cigarette in a holder and lit it. The system was primed by filling and emptying the syringe with smoke from the lit cigarette. Finally, $40 \mathrm{cc}$ of smoke was drawn from the lit cigarette into the syringe, after which the solenoid valve connecting the cigarette to the syringe was closed. Immediately thereafter, the subject was instructed to inhale smoke from the syringe, thereby drawing the barrel completely to the bottom of the syringe, delivering the entire $40 \mathrm{cc}$ of smoke to the subject. To make the procedure more natural, the subjects were allowed to inhale at their own rate. The subject then inhaled at least $500 \mathrm{cc}$ of air to wash the smoke from his mouth into his lungs, and after a variable period exhaled into a spirometer to confirm this minimum volume. The subjects were tested twice, with sessions at least a week apart. Breath carbon monoxide measures were taken before and after each testing session as an index of preceding smoking.

In the first practice session, the subjects inhaled two 40 -cc puffs of air from an unlit cigarette. The subjects rated the pleasantness/unpleasantness of the practice puff on a 10-point Likert scale and weakness or faintness on a 14-cm visual-analogue scale. This procedure was repeated twice as practice for the experimental procedure.

During the experimental procedure, the cigarette was lit and the subjects were instructed: "Continue smoking until you are no longer able to do so." The subjects rated each puff on two dimensions: (1) pleasantness/unpleasantness and (2) weakness and/or faintness. The subjects were instructed to follow the cigarette smoke by inhaling as large a volume of air as was comfortable and then exhaling into a spirometer. The experimenter confirmed that at least $500 \mathrm{cc}$ of air was inhaled following each puff. The subjects took one puff every $15 \mathrm{sec}$, signalled by an audiotaped instruction, with exactly 10 puffs per cigarette, which was then removed and extinguished. After each cigarette, there was a pause of $30 \mathrm{sec}$ to change cigarettes and to reprime the apparatus with smoke. The subjects smoked their own cigarette brand.

\section{RESULTS}

The mean difference between baseline Cambridge filter weight and postsmoke exposure weight for the Marlboro cigarettes was $4.95 \mathrm{mg}$, with a range of 4.7 to $5.3 \mathrm{mg}$. The standard deviation of the weights for the 10 trials was only $\pm 0.217 \mathrm{mg}$ or approximately $5 \%$, showing that the apparatus delivered reliable quantities of cigarette smoke as indicated by TPM.

In the second Cambridge filter study, Carlton ultralow nicotine/tar cigarettes were used. Using a procedure identical to that of the first Cambridge filter study, the mean weight difference for the 10 trials was $0.420 \mathrm{mg}$, with a range of 0.30 to $0.50 \mathrm{mg}$ and a standard deviation of $\pm .079 \mathrm{mg}$. The filtrate weights from regular and low cigarettes were significantly different $(p<.001)$. These results with a very low nicotine/tar-delivering cigarette indicated that this method and apparatus reliably differentiated high and low nicotine/tar cigarettes.

To examine the reliability of stop points in the controlled rapid-smoking procedure, the number of puffs to stopping in Trial 1 and Trial 2 were correlated and can be seen in Figure 2. The correlation between number of puffs taken in Trial 1 and Trial 2 was $.96(p<.001)$ with $N=8$. This indicates that the subjects reached approximately the same stop point on two different trials separated by a week. The mean carbon monoxide level before 
Distribution of Number of Puffs to End for Visit 1 versus Visit 2

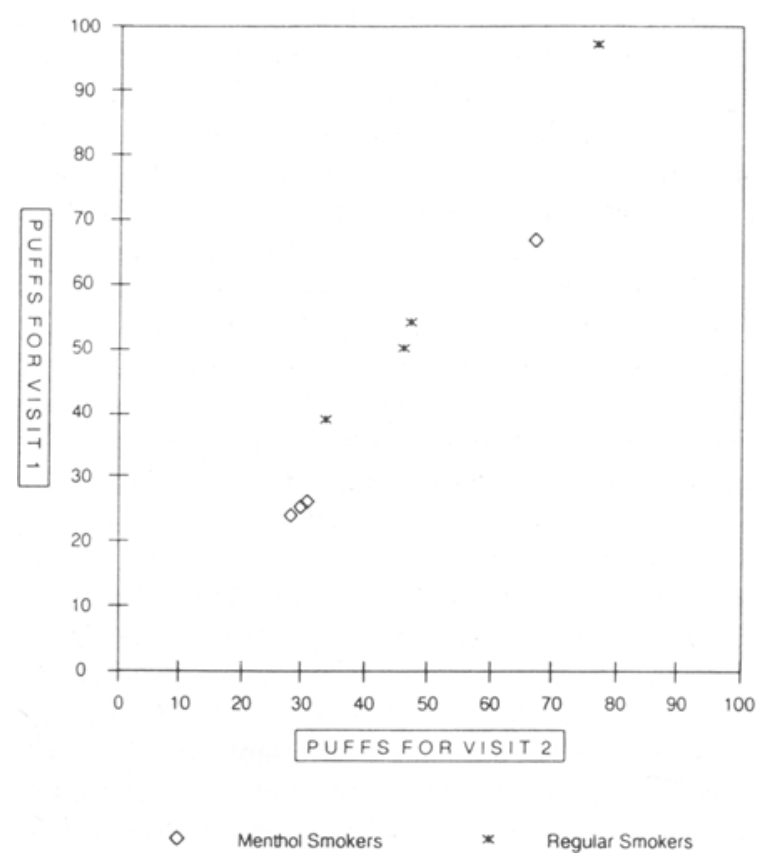

Figure 2. Scatter diagram of number of puffs to stop point by each subject for Visits 1 and 2.

the procedure $(M=21.1 \mathrm{ppm})$ was significantly different from the level after the procedure $(M=33.4 \mathrm{ppm})$ $(p<.001)$. Subjective ratings also changed from the beginning to the end of the procedure. The difference between mean pleasantness/unpleasantness for the first five puffs $(M=6.4)$ and the last five puffs $(M=3.3)$ was significant $(p<.001)$. Mean weakness/faintness for the first five puffs $(M=2.7 \mathrm{~cm})$ and the last five puffs $(M=$ $8.2 \mathrm{~cm})$ was also significantly different $(p<.001)$.

\section{DISCUSSION}

Results of the Cambridge filter studies indicated that the controlled smoke-delivery apparatus and procedure used in this study delivered reliable amounts of TPM from cigarette smoke that is readily accepted by smokers. Furthermore, the subjects exhibited reliable stopping points for the modified rapid-smoking procedure. The reliabil- ity of intrasubject stopping points demonstrated that the subjects could readily discriminate cues that signaled an unpleasant effect of nicotine. These cues are probably irritating stimuli from the upper respiratory tract plus aversive signals mediated by emetic and other protective centers in the brain. Thus, nicotine, though extremely potent, rarely brings smokers to emergency rooms.

\section{REFERENCES}

Benowitz, N. L., Jacob, P., III, Denaro, C., \&enkins, R. (1991). Stable isotope studies of nicotine kinetics and bioavailability. Clinical Pharmacology and Therapeutics, 49, 270-277.

Fagerstrom, K. O., \& SChNEIDER, N. G. (1989). Measuring nicotine dependence: A review of the Fagerstrom Tolerance Questionnaire. Journal of Behavioral Medicine, 12, 159-182.

Gilbert, D. G., Jensen, R. A., MeliskA, C. J. (1988). A system for administering quantified doses of tobacco smoke to human subjects: Plasma nicotine and filter pad validation. Pharmacology, Biochemistry \& Behavior, 31, 905-908.

Glasgow, R. E., Lichtenstein, E., Beaver, C., \& ONeill, K. (1981). Subjective reactions to rapid and normal paced aversive smoking. Addictive Behaviors, 6, 53-59.

Hatsukami, D., Keenan, R., Carroll, M., Colon, E., Geiske, D., WiLson, B., \& HuBer, M. (1990). A method for delivery of precise doses of smoked cocaine-base to humans. Pharmacology, Biochemistry \& Behavior, 36, 1-7.

Johanson, C. E., \& Fischman, M. W. (1989). The pharmacology of cocaine related to its abuse. Pharmacological Reviews, 41, 3-52.

Kozlowski, L. T., Herman, C. P. (1984). The interaction of psychosocial and biological determinants of tobacco use: More on the boundary model. Journal of Applied Psychology, 14, 244-256.

LEVIN, E. D., RosE, J. E., B BEHM, F. (1989). Controlling puff volume without disrupting smoking topography. Behavior Research Methods, Instruments, \& Computers, 21, 383-386.

Pomerleau, C. S., Majchrzak, M. J., \& Pomerleau, O. F. (1989). Paced puffing as a method for administering fixed doses of nicotine. Addictive Behaviors, 14, 571-575.

Pomerleau, O. F., Pomerleau, C. S., \& Rose, J. E. (1989). Controlled dosing of nicotine: A review of problems and progress. Annals of Behavioral Medicine, 11, 158-163.

Pomerleau, O. F., Rose, J. E., Pomerleau, C. S., \& Majchrzak, M. J. (1989). A noninvasive method for delivering controlled doses of nicotine via cigarette smoke. Behavior Research Methods, Instruments, \& Computers, 21, 598-602.

Rose, J. E., Tashkin, D. P., Ertle, A., Zinzer, M. C., \& Lafer, R. (1985). Sensory blockade of smoking satisfaction. Pharmacology, Biochemistry \& Behavior, 23, 289-293.

RUSSELL, M. A. (1989). Subjective and behavioural effects of nicotine in humans: Some sources of individual variation. Progress in Brain Research, 79, 289-302.

Sachs, D. P. L., Hall, R. G., Pechacek, T. F., \& Fitzgerald, J. (1979). Clarification of risk-benefit issues in rapid smoking. Journal of Consulting and Clinical Psychology, 47, 1053-1060.

(Manuscript received November 4, 1991; revision accepted for publication March 11, 1992.) 